\title{
Size matching in lung transplantation using predicted total lung capacity
}

\author{
J.P. Ouwens*, T.W. van der Mark*, W. van der Bij*, A. Geertsma*, W.J. de Boer", G.H. Koëter*
}

Size matching in lung transplantation using predicted total lung capacity. J.P. Ouwens, T.W. van der Mark, W. van der Bij, A. Geertsma, W.J. de Boer, G.H. Koëter. (C)ERS Journals Ltd 2002.

ABSTRACT: Height is used in allocation of donor lungs as an indirect estimate of thoracic size. Total lung capacity (TLC), determined by both height and sex, could be a more accurate functional estimation of thoracic size. Size-matching criteria based on height versus predicted TLC was retrospectively evaluated, and, furthermore, whether a TLC mismatch was related to clinical and functional complications.

The ratio of donor and recipient height, as well as the ratio of predicted TLC in donors and recipients, were calculated in 80 patients after bilateral lung transplantation. Complications evaluated included persistent atelectasis, persistent pneumothorax and increased number of days in intensive care, occurrence of bronchiolitis obliterans syndrome and limitation of exercise capacity.

Median height donor/recipient ratio was $1.01(0.93-1.12)$. Median predicted TLC donor/recipient ratio was 1.01 (with a clearly broader range $0.72-1.41$ ). Neither sex mismatch nor TLC mismatch were related to clinical or functional complications.

Allocation of donor lungs based upon height alone leads to a substantial mismatch in total lung capacity caused by sex mismatch. The absence of complications suggests that a greater height donor/recipient discrepancy can be accepted for allocation than previously assumed.

Eur Respir J 2002; 20: 1419-1422.
*Dept of Pulmonology, "Office for Medical Technology Assessment, and Dept of Thoracic Surgery, University Hospital Groningen, Groningen, the Netherlands.

Correspondence: J.P. Ouwens, Lung Transplantation Group, University Hospital Groningen, P.O. Box 30.001, 9700 RB Groningen, The Netherlands. Fax: 31503611738 E-mail: j.p.ouwens@mta.azg.nl

Keywords: Lung transplantation sex matching size matching total lung capacity

Received: November 92001 Accepted after revision: May 162002
Size matching in lung transplantation (LT) has been performed in the past by measuring the submammary thoracic perimeter $[1,2]$, by matching chest radiographs [3, 4], anthropometry, weight [5], predicted lung size [6] and height. However, none of these methods guarantee an appropriate lung size with respect to the size of the thoracic cage. The best approach to donor/recipient (D/R) size matching has not been determined so far.

In general, allocation of donor lungs is based primarily on blood group and height. Height mismatch may sometimes preclude allocation. The effect of the anatomical difference in shape of the thoracic cavity between males and females on the possible permitted height mismatch is unknown. Size matching based upon height alone may be associated with discrepancies in estimated thoracic size in case of a sex mismatch. A female recipient will receive a greater lung from a male donor although the height of donor and recipient is equal. Similarly, a male recipient will receive a smaller lung from a female donor although height of donor and recipient is equal. Total lung capacity (TLC) depends on height and sex and may thus be regarded as a more accurate, functional estimation of thoracic size.

In the present study, size matching based on height versus size matching based on height and sex were retrospectively evaluated by calculating ratios of $D / R$ height as well as ratios of predicted D/R TLC. Since an increased range of ratios of predicted TLC was expected, the authors investigated whether these size discrepancies led to clinical and/or functional complications.

\section{Methods}

\section{Patients}

Ninety-six bilateral LT (BLT) were performed between November 1990 and September 1998 in the Groningen University Hospital, Groningen, the Netherlands. The predicted TLC of all donors and recipients was calculated using the regression equations of the European Respiratory Society (ERS) [7, 8]. For males:

$$
\text { TLC predicted }=7.99 \mathrm{H}-7.08
$$

and for females:

$$
\text { TLC predicted }=6.60 \mathrm{H}-5.79
$$

where $\mathrm{H}$ represents height in $\mathrm{m}$.

These equations apply for persons of Caucasian descent, in the age range 18-70 yrs, and with a height of 1.55-1.95 m (males) or 1.45-1.80 m (females). 


\section{Size matching}

The degree of height matching was defined as the ratio of $\mathrm{D} / \mathrm{R}$ height. The degree of thoracic size matching was defined as the ratio of D/R TLC.

\section{Clinical and functional complications}

The range of predicted TLC is, by definition, determined by sex mismatching. Three cohorts of patients were defined according to the predicted TLC ratios: a sex-matched group, male to male and female to female (group 1); and two sex-mismatched groups, male to female (group 2) and female to male (group 3). Possible clinical complications of thoracic size mismatching were separated into short- and long-term complications. Short-term complications included an extended number of days in intensive care, persisting atelectasis and persisting pneumothorax. Atelectasis and persisting pneumothorax were defined as defects that originated on the first chest radiograph after LT and were still present after 1 week. Long-term clinical complications were defined as a decreased maximum exercise capacity 6 and 12 months after LT in a sexmismatched group, and early occurrence of bronchiolitis obliterans syndrome (BOS) compared to the sex-matched group. BOS grade 1 was defined as a persisting decline of forced expiratory volume in one second (FEV1) of $<80 \%$ of the mean of the two best FEV1 values, with or without changes in histology (i.e. grade $1 \mathrm{~A}$ or $1 \mathrm{~B}$ according to International Society for Heart and Lung Transplantation criteria for the classification of BOS [9]).

\section{Statistical analysis}

An increased occurrence of a persisting atelectasis in case of a male to female match (relatively larger lungs in a smaller thoracic cavity) and persisting pneumothorax in case of a female to male match (relatively smaller lungs in a larger thoracic cavity) were analysed by the Chi-squared test. The relationship between thoracic size mismatch and increased number of days in intensive care and decreased exercise capacity was calculated by one-way analysis of variance. Occurrence of BOS in the three sex(mis)matched groups was analysed using KaplanMeier survival estimates. A possible relationship between sex or TLC mismatching and early occurrence of BOS was investigated using the log-rank test. A p-value of $<0.05$ was considered statistically significant.
Table 1.-Patient characteristics

\begin{tabular}{lcc}
\hline & Donors & Recipients \\
\hline Patients n & 80 & 80 \\
Diagnosis & & \\
$\quad$ Bronchiectasis & & 7 \\
BOS & & 2 \\
COPD & 16 \\
Emphysema due to & \\
$\quad \quad 1$-antitrypsin & & \\
$\quad$ deficiency & & 29 \\
CF & & 16 \\
IPF & & 6 \\
$\quad$ Eisenmenger & & 4 \\
$\quad$ syndrome & $49: 31$ & $50: 30$ \\
Sex M:F & $36(18-54)$ & $45(20-64)$ \\
Age yrs & $1.75(1.55-1.95)$ & $1.74(1.55-1.94)$ \\
Height m &
\end{tabular}

Data are presented as median (range) unless otherwise stated. BOS: bronchiolitis obliterans syndrome; COPD: chronic obstructive pulmonary disease; CF: cystic fibrosis; IPF: idiopathic pulmonary fibrosis; M: male; F: female.

\section{Results}

Twelve patients with a donor age $<18$ yrs, two patients with a height outside the range, and two patients with a combination of age and height outside the ranges applicable for the regression equations of the ERS were excluded. Thus, 80 patients were included in the study (characteristics are given in table 1). Height and sex distributions for donors and recipients were similar. The median age of the recipient group was 9 yrs higher than the donor group, due to a relatively high percentage of patients with emphysema.

The median D/R height ratio was 1.01 (range 0.931.12; table 2). These ranges were centred around 1.0 in all four possible sex combinations. A similar observation was made for ranges based on predicted TLC for groups that were sex matched. However, in the groups with sex mismatch the D/R TLC ratio was $>1$ in the case of a male donor and a female recipient and $<1$ in the case of female donor and a male recipient. The median predicted $\mathrm{D} / \mathrm{R}$ TLC ratio was 1.01 (range $0.72-1.41$ ). This difference in range between the height ratio and the TLC ratio was therefore caused by sex mismatch.

Sex mismatch or TLC mismatch was not accompanied by significantly increased clinical or functional complications (table 3 ) nor differences in freedom from BOS ( $\mathrm{p}=0.590$; fig. 1). During transplantation, the size match seemed satisfactory in all patients. No mechanical difficulties were observed and no

Table 2. - Actually accepted height ratios and predicted total lung capacity (TLC) ratios for the different sex combinations

\begin{tabular}{lcccc}
\hline Donor to recipient & M to $\mathrm{M}$ & $\mathrm{F}$ to F & $\mathrm{M}$ to F & $\mathrm{F}$ to $\mathrm{M}$ \\
\hline Subjects $\mathrm{n}$ & 39 & 20 & 10 & 11 \\
Actually accepted range in D/R height ratio & $0.93-1.12$ & $0.96-1.10$ & $0.98-1.08$ & $0.93-1.03$ \\
Calculated range in D/R TLC ratio & $0.87-1.26$ & $0.92-1.23$ & $1.15-1.41$ & $0.72-0.89$ \\
\hline
\end{tabular}

M: male; F: female; D/R: donor/recipient. Note the differences in predicted TLC caused by sex mismatch. 
Table 3. - Comparison of clinical complications between the three cohorts

\begin{tabular}{|c|c|c|c|c|}
\hline Clinical or functional complication & $\mathrm{M}$ to $\mathrm{M}$ and $\mathrm{F}$ to $\mathrm{F}$ & $\mathrm{M}$ to $\mathrm{F}$ & $\mathrm{F}$ to $\mathrm{M}$ & p-value \\
\hline Cases $\mathrm{n}$ & 59 & 10 & 11 & \\
\hline With persisting atelectasis $>1$ week $\mathrm{n}(\%)$ & $5(8.5)$ & $0(0)$ & $0(0)$ & 0.373 \\
\hline With persisting pneumothorax $>1$ week $n(\%)$ & $4(6.8)$ & $1(10)$ & $0(0)$ & 0.592 \\
\hline Postoperative days on ICU & 11 & 19 & 15 & 0.088 \\
\hline Mean exercise capacity & 71 & 59 & 57 & 0.264 \\
\hline Mean exercise capacity Watt & 85 & 71 & 61 & 0.183 \\
\hline
\end{tabular}

M: male; F: female; ICU: intensive care unit. ${ }^{*}$ : 6 months after lung transplantation; ${ }^{\top}$ : 12 months after lung transplantation.

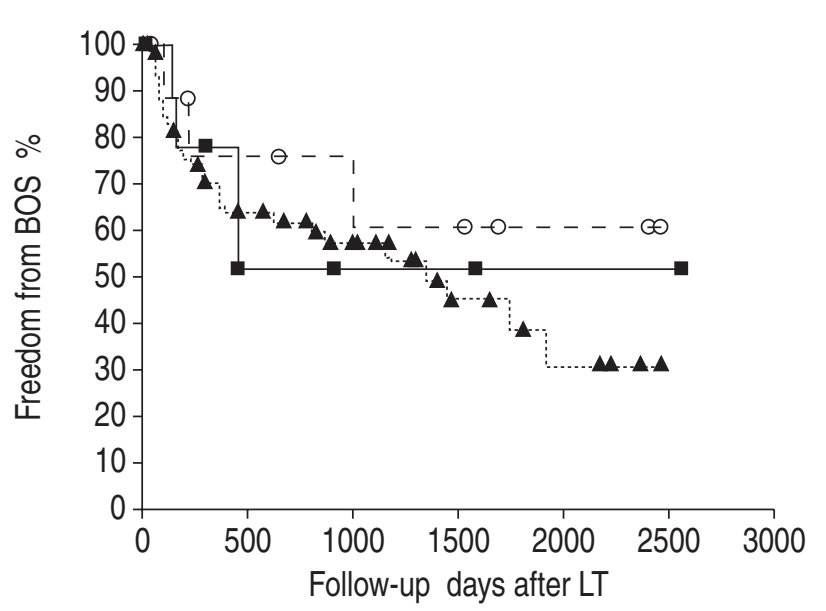

Fig. 1.-Sex (mis)match and freedom from bronchiolitis obliterans syndrome (BOS) after lung transplantation (LT). ----: male to female match; - : female to male match; …: same sex match. $\bigcirc$, $\boldsymbol{\square}, \boldsymbol{\Delta}$ : end of follow-up or patient died.

pneumoreduction procedures were performed during transplantation.

\section{Discussion}

This study shows that allocation of donor lungs based upon height leads to substantial mismatch in TLC, which is mainly caused by sex mismatch. This mismatch in TLC does not lead to early and late clinical and functional complications. The study showed that for precise size matching, allocation on height alone is not sufficient in cases of sex mismatch, but the absence of complications also suggests that a wider range of height matching could be possible in the sex-matched and sex-mismatched groups.

An accurate estimate of possible permitted height mismatch for the different sex combinations without occurrence of clinical and functional complications cannot be given. A study to explore possible permitted size mismatching more quantitatively would require more observations of complications. Such a study is clearly not possible. However, these data suggest that more leniency in height mismatching is possible, predominantly in the case of sex matching; in case of a male to female match a lower donor height and in case of a female to male match a higher donor height. Conversely, in the case of a male to female match, caution calls for a lower recipient (or higher donor) height, and in the case of a female to male match, a higher recipient (or lower donor) height.

Early experience suggests that a donor lung too large for the recipient's thoracic cavity would result in atelectasis and impaired ventilation, resulting in frequent infections [10]. Size matching is therefore limited and sometimes pneumoreduction by a surgical stapler is necessary in case of excess volume.

Outcome measures of size mismatching are hard to define. The early and late complications mentioned, such as pneumothorax, atelectasis, an increased number of intensive care days and exercise capacity, are determined by many factors. These parameters, however, are a reasonable choice to define size mismatch as outcome measures.

One of the investigated long-term functional complications was the possible relationship between the occurrence of BOS and TLC-mismatching or sex mismatch. This syndrome is believed to be an immunological process and not related to size matching. In a recent study, however, a relationship between sex mismatch and graft survival after LT was suggested [11]. In particular, male donors and female recipients had a poor prognosis with respect to graft function possibly caused by the difference in thoracic size between male donors and female recipients.

The fact that no differences in clinical complications were found between the three sex-(mis)matched groups must be interpreted with caution. In descriptive studies, as presented here, it is not always possible to choose the optimum group size for avoiding a type II error. However, with the given group size, a difference of $30 \%$ in occurrence of complications would have been detected.

Allocation using the predicted D/R TLC was studied as a concept in patients after BLT. The value of this method and the possible range in TLC for single LT recipients must be studied in the future. Exceptions for using the predicted TLC are recipients with a restrictive lung disease. In these patients, donor lungs should be selected with great caution because of the small thoracic cavity of the recipient due to their underlying disease. In practice, this means that height of the donor should not exceed height of the recipient. Parenchymal injury, obstruction, repeated infections and even pneumoreduction could be a consequence.

The regression equations used in this study apply to a certain range in height, age and Caucasian race. Predicted values for TLC in children $>5$ yrs of age can be calculated using special regression equations 
developed for children based on TLC-helium measurements [12]. Conversion factors for calculating predicted values in people of other ethnic groups for adjusting European reference values are available [13]. The predicted lung size of a person of other or mixed racial origin is intermediate between that of the parents [14].

Due to donor organ shortage, most lung transplantation centres have long waiting lists. Therefore, donor lungs can usually be allocated. However, in the case of a special blood group, special height or seriously ill patients on a special urgency waiting list, height mismatch may preclude proper allocation. This study suggests that increased ranges in height for allocation of donor lungs can be permitted. This may have an impact on the allocation of donor lungs for patients with a special blood group, special height or an urgency.

\section{References}

1. Noirclerc M, Shennib H, Giudicelli R, et al. Size matching in lung transplantation. $J$ Heart Lung Transplant 1992; 11: S203-S208.

2. Massard G, Badier M, Guillot C, et al. Lung size matching for double lung transplantation based on the submammary thoracic perimeter. Accuracy and functional results. The Joint Marseille-Montreal Lung Transplant Program. J Thorac Cardiovasc Surg 1993; 105: 9-14.

3. Hakim M, Higenbottam TW, Bethune D, et al. Selection and procurement of combined heart and lung grafts for transplantation. $J$ Thorac Cardiovasc Surg 1988; 95: 474-479.

4. Harjula A, Baldwin JC, Starnes VA, et al. Proper donor selection for heart-lung transplantation. The Stanford experience. J Thorac Cardiovasc Surg 1987; 94: 874-880.
5. Griffith BP, Hardesty RL, Trento A, et al. Heart-lung transplantation: lessons learned and future hopes. Ann Thorac Surg 1987; 43: 6-16.

6. Miyoshi S, Schaefers HJ, Trulock EP, et al. Donor selection for single and double lung transplantation: chest size matching and other factors influencing post transplantation vital capacity. Chest 1990; 98: 308313.

7. Roberts CM, Macrae KD, Winning AJ, Adams L, Seed WA. Reference values and prediction equations for normal lung function in a non-smoking white urban population. Thorax 1991; 46: 643-650.

8. Ghio AJ, Crapo RO, Elliot CG. Reference equations used to predict pulmonary function. Survey at institutions with respiratory disease training programs in the United States and Canada. Chest 1990; 97: 400-403.

9. Cooper JD, Billingham M, Egan T, et al. A working formulation for the standardization of nomenclature and for clinical staging of chronic dysfunction in lung allografts. International Society for Heart and Lung Transplantation. J Heart Lung Transplant 1993; 12: 713-716.

10. Egan TM, Thompson JT, Detterbeck FC, et al. Effect of size (mis)matching in clinical double-lung transplantation. Transplantation 1995; 59: 707-713.

11. Love RB, Meyer KC, Devito-Haynes LD, et al. Effect of HLA-DR mismatch on lung transplant outcome. J Heart Lung Transplant 2001; 20: 177.

12. Cook CD, Hamann JF. Relation of lung volumes to height in healthy persons between the ages of 5 and 38 years. J Paediatr 1961; 59: 710-714.

13. Cotes JE. Lung function: Assessment and application in medicine. Oxford, Blackwell Scientific Publications, 1993.

14. Miller GJ, Saunders MJ, Gilson RJC, Ashcroft MT. Lung function of healthy boys and girls in Jamaica in relation to ethnic composition, test exercise performance and habitual physical activity. Thorax 1977; 32 : 486-496. 